\title{
Social distance regulation for control of COVID-19 may foster the global obesity pandemic: Social distancing increases the desire for food
}

\section{Yevvon Yi-Chi Chang}

Tunghai University

Wen-Hsiung Wu

Kaohsiung Medical University

Wen-Bin Chiou ( $\sim$ wbchiou@mail.nsysu.edu.tw )

National Sun Yat-sen University

\section{Research Article}

Keywords: COVID-19, desire for food, social distance regulation, social distancing, substitutability

Posted Date: September 21st, 2020

DOI: https://doi.org/10.21203/rs.3.rs-80438/v1

License: (c) (i) This work is licensed under a Creative Commons Attribution 4.0 International License.

Read Full License 
Title Page

Social distance regulation for control of COVID-19 may foster the global obesity pandemic: Social distancing increases the desire for food

Yevvon Yi-Chi Chang ${ }^{1}$, Wen-Hsiung $\mathrm{Wu}^{2} \&$ Wen-Bin $\mathrm{Chiou}^{3 *}$

${ }^{1}$ Department of Hospitality Management, Tunghai University, Taichung 407224, Taiwan.

${ }^{2}$ Department of Healthcare Administration and Medical Informatics, Kaohsiung Medical University, Kaohsiung 80708, Taiwan. ${ }^{3}$ Institute of Education, National Sun Yat-sen

University, Kaohsiung 80424, Taiwan. *email: wbchiou@mail.nsysu.edu.tw

\section{Acknowledgements}

This work was partially supported by a grant funded by Ministry of Science and Technology, Taiwan (MOST 109-2410-H-110-055-MY3).

\section{Author contributions}

Y. Y. C., W. W. and W. C. planned the experiment. Y. Y. C. performed the experiment. Y. Y. C. and W. W. analyzed the data. W. W. and W. C. wrote the draft of the manuscript. All authors have edited the manuscript.

\section{Competing interests}

The authors declare no competing interests. 
Social distance regulation for control of COVID-19 may foster the global obesity pandemic: Social distancing increases the desire for food

Social distance regulations have been suggested as one of the best ways to control and prevent the spread of coronavirus pneumonia (COVID-19). Social connection and food are intertwined because both have played critical evolutionary roles in human survival. We tested whether the substitutability hypothesis in human motivation applies here in that cues signaling scarcity in one domain (e.g., social connection) might enhance the desire to acquire resources in another domain (e.g., food). In a laboratory experiment $(N=118)$, we found that, compared with controls, participants primed with social distancing consumed more ice cream in a taste test and reported a greater likelihood that they would engage in binge eating if they were placed in home quarantine. We may be the first to provide experimental evidence that social distancing can enhance the desire for food. The link between social distancing and the desire for food is pertinent to understanding how strongly social distance regulations may influence weight gain. Our findings have far-reaching implications for weight control under social distance regulations for prevention and control of COVID-19. 
To prevent and control the spread of coronavirus pneumonia (COVID-19), social distance regulations have been implemented in many countries ${ }^{1}$. However, humans rely on one another to obtain what they want and need ${ }^{2,3}$, and this social interdependency nourishes a strong desire for social connection ${ }^{4}$. Evolutionary psychologists argue that the desire to form and maintain social bonds has an evolutionary basis ${ }^{5,6}$. Additionally, from an evolutionary perspective, one of the primary challenges for human survival is to collect or produce enough food $^{7,8}$. Hence, both food $^{7}$ and social connection ${ }^{3}$ should be considered as subsistence resources for human survival.

Given that social connection is important to human survival, a meaningful life, and well-being ${ }^{9}$, it is crucial for people to monitor the abundance or scarcity of social connection. According to the fundamental principle of self-regulation of motivation and action ${ }^{10}$, cues that signal deprivation (abundance) of one of the subsistence resources should increase (decrease) the incentive value of others ${ }^{11}$. Some behavioral evidence supports this substitutability hypothesis ${ }^{2}$. For example, Briers et al. ${ }^{11}$ showed that hungry participants were less likely to make monetary donations than were satiated participants. Zhou, Vohs, and Baumeister ${ }^{12}$ demonstrated that participants under a social rejection condition donated less money than those under a social acceptance condition. Lasaleta et al. ${ }^{3}$ reported that a sense that social connection was plentiful decreased the desire for money.

According to the principle of the substitutability of subsistence resources ${ }^{2,11}$, cues signaling scarcity in one subsistence resource should motivate people to acquire other subsistence resources. However, little research has empirically tested whether experiencing scarcity in social connection (i.e., social distancing) increases the desire for food. The current study was aimed at rectifying this deficit in the research literature. Therefore, our investigation examined the hypothesis that when people feel that social connection with 
others is scarce, their desire for food increases. We contend that priming with social distancing may enhance the desire for food, especially under social distance regulations intended to deal with the threat of COVID-19. We predicted that people who are reminded of social distancing (i.e., scarcity in social connection) may experience an increase in the desire for food, as manifested by their eating more ice cream in a taste test and reporting greater likelihood of binge eating.

\section{Results and discussion}

Table 1. The desire for food as a function of felt social disconnection and social distancing.

Measures

Experimental condition

\begin{tabular}{llll}
\hline \multicolumn{2}{l}{ Social distancing } & \multicolumn{2}{c}{ Control } \\
\hline Mean $95 \% \mathrm{CI}$ & Mean & $95 \% \mathrm{CI}$
\end{tabular}

Intention to lose weight (1-5)

2.93

$[2.64,3.23]$

2.80

$[2.53,3.06]$

Time since last meal (hr)

1.76

$[1.63,1.90]$

1.71

$[1.57,1.86]$

Participant BMI $\left(\mathrm{kg} / \mathrm{m}^{2}\right)$

23.32

[22.74, 23.91]

22.70

Felt social disconnection (1-7)

4.93

$[4.55,5.32]$

3.98

Amount of ice cream eaten $(\mathrm{g})$

$151.49 \quad[145.81,157.17]$

$137.81 \quad[132.35,143.28]$

Likelihood of binge eating (1-7)

4.48

$[4.12,4.83]$

3.60

[3.21, 3.97]

Note: Units of the dependent measure are presented in parentheses. $\mathrm{CI}=$ confidence interval. $\mathrm{BMI}=$ body mass index 
The intention to lose weight $(t(116)=0.687, p=0.493)$, the time since the last meal $(t(116)=$ $0.516, p=0.607)$, and participant BMI $(t(116)=1.58, p=0.117)$ did not differ between the social distancing and control conditions (see Table 1), indicating that the random assignment created equivalent groups. In addition, the amount of ice cream eaten was not associated with the intention to lose weight $(r=-0.14, p=0.13)$, the time since the last meal $(r=0.097, p=$ 0.297), and participant BMI $(r=0.064, p=0.494)$. The self-reported likelihood of binge eating was not related to the intention to lose weight $(r=-0.122, p=0.189)$, the time since the last meal $(r=0.153, p=0.099)$, and participant BMI $(r=0.056, p=0.547)$. Hence, these three factors were not treated as control variables in subsequent analyses.

Table 1 shows that participants receiving the social distancing prime felt more socially disconnected $(M=4.93, S D=1.47)$ than did those receiving the neutral prime $(M=3.98, S D$ $=1.29), t(116)=3.721, p<0.001$, Cohen's $d=0.69$. As intended, participants in the social distancing condition ate more ice cream in the taste test $(M=151.49 \mathrm{~g}, S D=21.79)$ than did those in the control condition $(M=137.81 \mathrm{~g}, S D=20.96), t(116)=3.475, p<0.001, d=0.64$. The effect of social distancing on the desire for food was also evidenced by the reported likelihood of binge eating. Participants primed with social distancing were, by self-report, more likely to engage in binge eating if they were placed in home quarantine $(M=4.47, S D=$ 1.37) than were control participants $(M=3.59, S D=1.45), t(116)=3.393, p<0.001, d=$ 0.62. Furthermore, participants' ratings of felt social disconnection were associated with the amount of ice cream consumed in the taste test $(r=0.558, p<0.001)$ and with a higher reported likelihood of binge eating $(r=0.533, p<0.001)$. Additionally, the interaction between participant sex and social distancing had no significant effect on the amount of ice cream eaten in the taste test or the likelihood of binge eating $(F s<1, p s>0.05)$. 


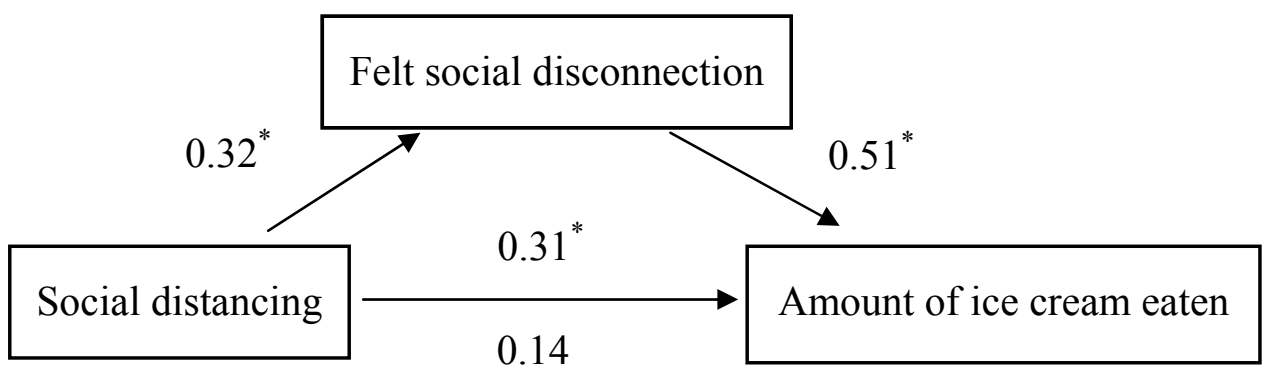

Figure 1. Mediation of the effect of social distancing on the amount of ice cream eaten in a taste test. Values are standardized regression coefficients. On the lower path, the values below and above the arrow are the results of analyses in which the mediator (felt social disconnection) was and was not included in the model, respectively. An asterisk indicates a $p$-value of less than 0.01 .

The mediating role of felt social disconnection was also tested against our two indicators of desire for food. The experimental condition was dummy coded $(1=$ social distancing, $0=$ control). The effect of social distancing on the amount of ice cream eaten in the taste test $(B=$ $13.68, S E=3.94, t=3.475, p<0.001)$ was not significant $(B=6.22, S E=3.60, t=1.728, p>$ 0.05) after controlling for felt social disconnection. The $95 \%$ bias-corrected confidence interval $(\mathrm{CI} ; 3.55$ to 12.62$)$ for the indirect effect $(B=7.52, S E=2.27$; bootstrap resamples $=$ 5,000 ) was significant in a bootstrap analysis ${ }^{13}$, indicating that felt social disconnection played a mediating role (See Figure 1). The mediating effect of feeling socially disconnected was also observed regarding the likelihood of binge eating. When felt social disconnection was controlled, the relationship between social distancing and the likelihood of binge eating $(B=0.88, S E=0.26, t=3.393, p<0.001)$ was not significant $(B=0.41, S E=0.24, t=1.716$, $p>0.05)$. Furthermore, the indirect effect of felt social disconnection $(B=0.47, S E=0.16$, 
95\% bias-corrected CI: 0.20 to 0.84 ; bootstrap resamples $=5,000)$ was significant, indicating that felt social disconnection mediated the effect of social distancing on participants' likelihood of binge eating.

Building on the notion that social distancing may signify scarcity in one subsistence resource and thereby motivate people to acquire other subsistence resources (e.g., food), we conducted a laboratory experiment to test the possibility that participants primed with social distancing would eat more ice cream in a taste test and would be more likely to engage in binge eating. The present findings indicate an asymmetric relation between the desire for social connection and the desire for food. To our knowledge, we may be the first to provide experimental evidence that confirms the psychological effect of social distancing on the desire for food.

Our findings contribute to the literature in several important ways. The observed link between social distancing and the desire for food provides support to the substitutability principle regarding human motivation regulation ${ }^{2,10}$. When one subsistence source is seen as scarce, people are motivated to pursue another. We found that social distancing increased the desire for food, in keeping with the theory that social connectedness and food operate as entwined subsistence resources. Furthermore, the present findings are in line with prior research on the reciprocal substitutability of social connection and money. For example, three experiments conducted by Lasaleta and Vohs ${ }^{2}$ showed that social support primes a decreased desire for money, as manifested by ranking financial success as less important, reporting less worry over money, and seeing money as less important. Twenge, Baumeister, DeWall, Ciarocco, and Bartels ${ }^{14}$ found that participants who were led to believe that they were likely to be alone later in life donated less money than did participants led to expect future belonging and the control group (Experiment 1). In addition, Zhou et al. ${ }^{12}$ demonstrated that participants in the social rejection group donated less money than those in the social 
acceptance group. Just as priming with money can buffer the impact of social exclusion by making one feel strong, the current research indicates that social distancing may increase the desire for food by making one feel less socially connected. Finally, part of our contribution lies in providing a viable explanation for a prevalent phenomenon under the impact of COVID-19, namely, that many people compulsively hoard materials such as food, face masks, and everyday necessities like toilet paper.

With regard to limitations and future directions, the present research employed the amount of ice cream eaten in a taste test and self-reported likelihood of binge eating as indicators of a desire for food. Use of other measures such as daily food consumption or weight gain may support the practical and external validity of this approach. Prior research has shown that money and food are entwined and interchangeable ${ }^{11,15,16}$, and future research may test whether social distancing might also promote the desire for money. We observed that priming with social distancing induced a heightened desire for food. The question of whether socially disconnected people are vulnerable to overeating and obesity-related problems is worthy of investigation. Finally, an important area for future research is the overlap in neurological activation between the incentive value of social connection and that of food. Neurological evidence showing that these two reward systems share a common brain region has important implications for weight control and interventions using comfort food.

\section{Methods}

Participants. A total of 118 undergraduate and graduate students (62 females; mean age = 21.4 years, $S D=1.9)$ were recruited to participate in this experiment. During recruitment, the participants were screened to meet the following criteria: no eating disorders, no dieting, no current weight reduction therapy, and not taking weight reduction supplements. The sample size was estimated by calculating the number of participants required to test a directional 
hypothesis regarding the mean difference between two independent groups under the following conditions: $\alpha=0.05, d=0.50$ (medium effect $\left.\operatorname{siz} \mathrm{e}^{17}\right)$, and power $=0.85$. This study was approved by the IRB of Kaohsiung Medical University. The informed consent was obtained from all participants.

Procedure. Upon arrival, participants were told that they were helping to test unrelated tasks that would be used in future studies. After providing consent, participants reported the time since their last meal (in hours) and rated their intention to lose weight on a five-point scale $(1=$ very low, 5 = very high $)$.

By employing block randomization schedules, every two participants were assigned to one of the two study conditions (social distancing vs. control). We employed the emotional-event recollection technique ${ }^{18,19}$ to prime experiences of social distancing. For the social distancing condition, participants were asked to recall a salient and impressive event that made them feel a strong sense of social disconnection due to the government's social distance regulations around COVID-19. For the control condition, participants were asked to recall a routine event from before the COVID-19 pandemic. After the recall task, all participants were asked to rate their felt social disconnection in their immediate experience on a 7 -point scale $(1=$ not at all, $7=$ very much $)$. In a pretest $(N=56 ; 30$ females $)$ conducted to check the effectiveness of our manipulation, participants in the social distancing condition reported a higher level of felt social disconnection $(M=4.79, S D=1.50)$ than those in the control condition $(M=3.75, S D=1.51 ; t(54)=2.579, p=0.013, d=0.75)$. These results confirmed that our manipulation affected immediate feelings of social disconnection.

Later, participants were asked to complete a filler questionnaire while the experimenter prepared the taste test. The short survey included a question assessing the sense of social disconnection $(1=$ not at all, $7=$ very $m u c h)$. For the taste test, we provided participants with 
two different flavors of ice cream (each $\sim 200 \mathrm{~g}$, equivalent to $400 \mathrm{kcal}$ ). To disguise the real purpose of the study, participants were asked to compare these two ice creams and complete a sensory evaluation questionnaire. In the demographic section of that questionnaire, we embedded an item to measure the likelihood of binge eating in a social disconnection situation ("If you had to be placed in home quarantine, how likely would you be to engage in binge eating?"). Participants answered the question using a scale from 1 (very unlikely) to 7 (very likely). The amount of ice cream consumed and the self-rated likelihood of binge eating were used as the dependent measures. In addition, self-reported height and weight were used to calculate the body mass index $\left(\mathrm{BMI} ; \mathrm{kg} / \mathrm{m}^{2}\right)$. At the end of this experiment, participants were probed to examine whether they had suspected the true purpose of the study. None of them guessed the true goals of this experiment.

\section{Conclusions}

The current research indicates that merely reminding people of social distancing, without reaching an intense level of ostracism, may promote the desire for food. Social distance regulations have been widely adopted for prevention and control of coronavirus disease. Our findings suggest that social distancing may increase the desire for food, so social distance regulations may be more closely associated with weight gain or even with an obesity pandemic than previously thought. Perhaps the impact of social distancing under COVID-19 regulations is so powerful that individuals feel less socially connected and feel that way more often. Accordingly, to the degree that social connection and food are interchangeable subsistence resources, people might consume more food to appease their social pain, thereby sacrificing their health. The impact of social distance regulations during and after the global pandemic on humans' striving for food should receive more attention than it presently does. 


\section{Data availability}

The dataset generated during and/or analyzed during the current study are available on the Open Science Framework repository (https://osf.io/et94f/).

All methods were performed in accordance with the relevant guidelines and regulations.

\section{References}

1. Parmet, W. E. \& Sinha, M. S. Covid-19: The law and limits of quarantine. N. Engl. J. Med. 382, e28 (2020).

2. Lasaleta, J. D. \& Vohs, K. D. With friends like these who needs money? Three tests of the substitutability hypothesis of money and social support. Adv. Consum. Res. 41, 191-192 (2013).

3. Lasaleta, J. D., Sedikides, C. \& Vohs, K. D. Nostalgia weakens the desire for money. J. Consum. Res. 41, 713-729 (2014).

4. Baumeister, R. F. \& Leary, M. R. The need to belong: Desire for interpersonal attachments as a fundamental human motivation. Psychol. Bull. 117, 497-529 (1995).

5. Ainsworth, M. S. Attachments beyond infancy. Am. Psychol. 44, 709-716 (1989).

6. Buss, D. M. Evolutionary personality psychology. Annu. Rev. Psychol. 42, 459-491 (1991).

7. Diamond, J. Guns, germs, and steel: The fates of human societies (Norton, 1997).

8. Briga, M., Koetsier, E., Boonekamp, J. J., Jimeno, B. \& Verhulst, S. Food availability affects adult survival trajectories depending on early developmental conditions. Proc. Royal Soc. B 284, 20162287 (2017).

9. Stavrova, O. \& Luhmann, M. Social connectedness as a source and consequence of 
meaning in life. J. Posit. Psychol. 11, 470-479 (2016).

10. Carver, C. S. Self-regulation of action and affect in Handbook of self-regulation: Research, theory, and applications (eds. Baumeister, R. F, Vohs, K. D.) 13-39 (Guilford, 2004).

11. Briers, B., Pandelaere, M., Dewitte, S. \& Warlop, L. Hungry for money: The desire for caloric resources increases the desire for financial resources and vice versa. Psychol. Sci. 17, 939-943 (2006).

12. Zhou, X., Vohs, K. D. \& Baumeister, R. F. The symbolic power of money: Reminders of money alter social distress and physical pain. Psychol. Sci. 20, 700-706 (2009).

13. Preacher, K. J., \& Hayes, A. F. SPSS and SAS procedures for estimating indirect effects in simple mediation models. Behav. Res. Methods Instrum. Comput. 36, 717-731 (2004).

14. Twenge, J. M., Baumeister, R. F., DeWall, C. N., Ciarocco, N. J. \& Bartels, J. M. Social exclusion decreases prosocial behavior. J. Pers. Soc. Psychol. 92, 56-66 (2007).

15. Nelson, L. D. \& Morrison, E. L. The symptoms of resource scarcity: Judgments of food and finances influence preferences for potential partners. Psychol. Sci. 16, 167-173 (2005).

16. Pettijohn, T. F. \& Jungeberg, B. J. Playboy playmate curves: Changes in facial and body feature preferences across social and economic conditions. Pers. Soc. Psychol. Bull. 30, 1186-1197 (2004).

17. Cohen, J. Statistical power analysis for the behavioral sciences (Erlbaum, 1988).

18. Chao, Y., Cheng, Y. \& Chiou, W. The psychological consequence of experiencing shame: Self-sufficiency and mood-repair. Motiv. Emot. 35, 202-210 (2011).

19. Leith, K. P. \& Baumeister, R. F. Why do bad moods increase self-defeating behavior? Emotion, risking-taking, and self-regulation. J. Pers. Soc. Psychol. 71, 1250-1267 (1996). 
Figures

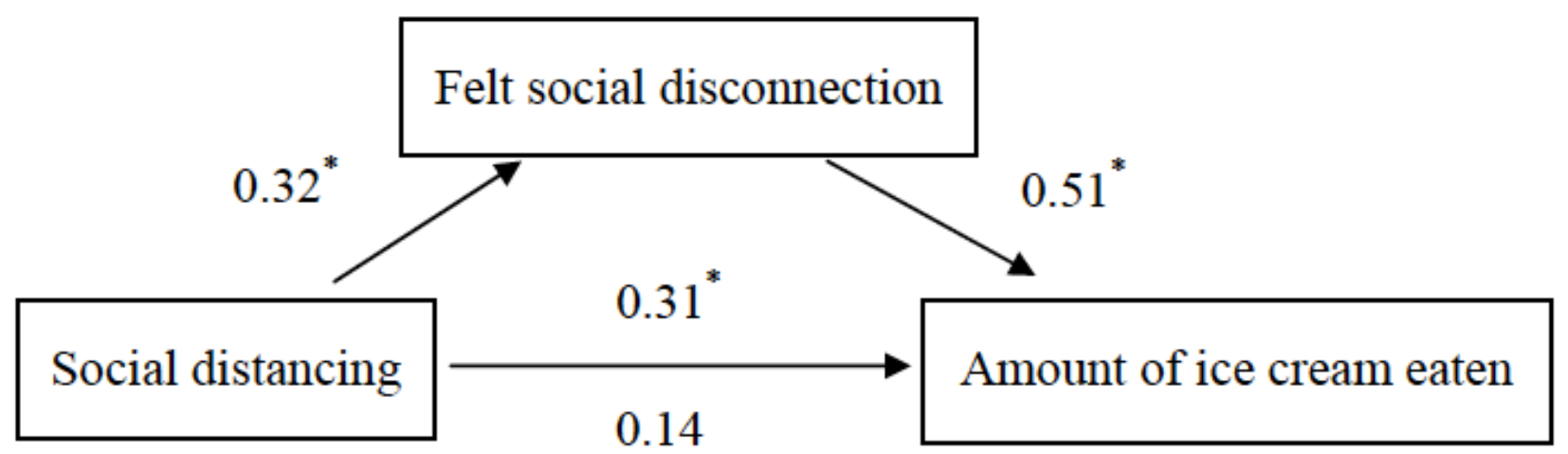

Figure 1

Mediation of the effect of social distancing on the amount of ice cream eaten in a taste test. Values are standardized regression coefficients. On the lower path, the values below and above the arrow are the results of analyses in which the mediator (felt social disconnection) was and was not included in the model, respectively. An asterisk indicates a p-value of less than 0.01 . 